\title{
An Analysis of the Possibility of Origami Implementation in Mathematics Learning Process in Indonesia
}

\author{
Muhammad Toyib, NaufalIshartono \\ Mathematics Education Department \\ Universitas Muhammadiyah Surakarta \\ Surakarta, Indonesia \\ muhamad.toyib@ums.ac.id
}

\begin{abstract}
Origami is the art of paper folding originated from Japan. It is, based on previous research has many benefits, especially in the learning of mathematics in the classroom. Therefore, there is a need to study about the possibility of application of origami in the process of learning mathematics in Indonesia. This study aimed to find out and analyze the possibility of application of origami in the Indonesian education system, especially in the subjects of mathematics at junior high school. This research is the first (first year) research within two phases (2 years) of research. The research method was done by a means of surveying technique, by spreading the questionnaire to the junior high school mathematics teachers via Google Spreadsheet, the analysis of BSE mathematics junior high school books, and discussion with some mathematics teachers. The result of this research shows that there is a huge chance for origami to be applied in mathematics learning process in junior high school level in Indonesia because based on the result of the questionnaire, $87.5 \%$ of respondents stated that they have never used origami as learning media. In addition, from the results of the analysis of 6 mathematics BSE books of junior high school level in Indonesia, it was found that origami or paper folding art is only applied to one activity and become an example of the origami-based problem. It is concluded that the development of secondary-school mathematics learning model by using origami has a huge opportunity to be implemented.
\end{abstract}

Keywords: Origami; Mathematical Learning; Junior High School Mathematics

\section{INTRODUCTION}

Mastery of mathematics becomes an obligatory that must be mastered by the global community today. Many research results indicate that the mastery of mathematics provides convenience to someone in solving problems in their daily lives. As a result of Aiken's research [1] mathematics helps one's thinking ability and train them to think. While the results of research by Peters [2] states that "knowing how to use the numbers is important for everyone, even if you think you're not using math, Numeracy helps people in their everyday lives". Therefore, the role of learning mathematics in schools has a very important role in improving understanding and mastery of mathematics.

In Indonesia, the level of understanding of mathematics for junior high school students is still need to be improved. Based on the results of the latest PISA (Program of International Student Assessment) and TIMSS (Trend in Mathematics and Science Study) that reports sequentially, it is showed that the math literacy rate for Indonesian students is at the $63^{\text {rd }}$ position of 69 PISA participating countries (www.oecd.org/ Pisa /) and the $36^{\text {th }}$ position of 49 participating countries TIMSS (https://nces.ed.gov/timss/). Of course, the report can be used as a reference for academics and education practitioners, especially in the field of mathematics in making a more comprehensive learning innovation. One of them is by developing learning media.

One of the learning innovations that can improve students' understanding of mathematics is the use of origami in mathematics learning. According to Hull [3], "origami is the art of folding paper without the aid of scissors or glue, which is formed into pieces of sculptures". Origami is very easy and safe to use as a medium for learning mathematics for students, so the combination between learning mathematics and origami games will make the learning process becomes more enjoyable, such as the result from Gur\&Kobak's research [4]. In addition, the results of the study also suggest that the use of origami is very effective in improving students 'spatial thinking skills, students' cognitive abilities [6], concept reinforcement (Cipoletti\& Wilson in Boakes [7]), and students' interest in mathematics (Pearl in Boakes [7]).

Based on the many benefits derived from the use of origami in mathematics learning, the authors believe that origami will help mathematics teachers to improve the quality of mathematics teaching-learning process in Indonesia. Therefore, the authors will develop mathematics instructional devices based on origami that will be done in 2 years research. However, the authors need to find out whether there is any possibility to implement origami in mathematics classes in the Indonesian school. Thus, this study aims to analyze the possible use of origami in the junior high school mathematics learning process in Indonesia.

\section{RESEARCH METHOD}

The research subjects are junior high school mathematics teachers in Indonesia. The data were collected using four methods: 1) through teacher questionnaire that was distributed using Google Spreadsheet, 2) interview with the teacher, 3) literature analysis, and 4) analysis of students' textbook.

Questionnaires are used to find out how many teachers have used origami as media to teach mathematics. Teacher discussions were used to find out the teacher's point of view of how important is the use of origami as media to teach mathematics at school and to know the opinions and inputs of 
the developed prototype. Literature analysis is used to find out the application of origami in mathematical learning theoretically that based on sources of journal and book, and what kind of learning method that can be integrated with origami. While students' textbook analysis is used to find data about how far origami is used as an example or activity in a students' textbook, and if it exists, on what topic are origami used as the basis of activity in the students' textbook.

The data were analyzed according to the type of data collection method. For the questionnaire, the data were analyzed quantitatively by using the proportion method. While for the three other data collecting methods, they were analyzed by using the qualitative method.

\section{RESULT AND DISCUSSION \\ A. Data on the Use of Origami as a Mathematics Learning Media in Indonesia}

The effort to search data of origami usage in mathematics learning process in Indonesia was done by distributing questionnaires to several teachers in various places in Indonesia. The questionnaire is developed by utilizing Google Spreadsheet which contains questions that are broadly asked about the experience of respondents in using origami in the learning process of mathematics that has been done. It also asks the lack and the advantages of using origami as a medium of learning mathematics, and asks teachers' perception about the use of origami as a medium of learning mathematics.

Based on the questionnaire, the data were obtained as follows:

1. From $100 \%$ of incoming data obtained, only $12.5 \%$ of respondents used origami as a medium for learning mathematics.

2. From a number of respondents who have used origami in the classroom learning, it is stated that learning becomes more fun and can increase students' enthusiasm in learning mathematics.

3. While a number of respondents who have never used origami, it is stated that origami is very likely to be used as a medium for learning mathematics.

Based on the results of the questionnaire analysis it is concluded that there are still very few mathematics teachers who apply origami as a medium for learning mathematics. This suggests that there is still a very high probability of using origami in mathematics learning in schools in Indonesia.

\section{B. Literature Analysis}

The literature analysis is used to find out the topics of mathematics in junior high school that can be integrated with origami, criteria of good teaching materials, and learning models that can be integrated with origami.

The steps for determining mathematical topics that can be integrated with origami began with analyzing the student's mathematical handbooks. The books analyzed are Electronic School Books (BSE) produced by the Ministry of Education and Culture (Kemdikbud) because this book becomes a standard book used as a reference in classroom learning. The result of BSE book analysis found that from 6 BSE mathematics books (7th, 8th and 9th grade), origami is only applied to one activity. It is in BSE Mathematics, grade 8 the Year 2017 Semester II in Activity 7.2.: Determine the relationship between the center angle with, and one example that is in BSE Mathematics grade 7 Year 2017 Semester II in Example 8.23: Area of triangle. Therefore, there are still many opportunities for origami to be developed and integrated into mathematics topics.

The topics that can be integrated with origami as learning media are as follows:

Table 1. Mathematical Topics that Can Be Integrated with Origami

\begin{tabular}{|c|l|}
\hline No. & \multicolumn{1}{|c|}{ Topic } \\
\hline 1. & Integer operation, arithmetic numbers, series of number \\
\hline 2. & Elements and properties plane (two-dimension) \\
\hline 3. & Elements and properties of space (three-dimension) \\
\hline 4. & Statistical data presentation \\
\hline 5. & The concept of probability theories \\
\hline
\end{tabular}

Of course, there is a need for a learning model that can engage students actively during the learning process and can be integrated with origami. The learning model that fits the purpose is project-based learning (PjBL). Project-based learning $(\mathrm{PjBL})$ is a model that organizes learning around projects [9]. Project-based learning refers to the theory and practice of utilizing objectives and to facilitate individual and collective learning [10]. In general, the goals in problem-based learning are twofold [11]: 1) to promote deep understanding of subject matter content, and 2) to simultaneously develop higher-order thinking skills. The characteristics of PjBL (Markham et al. In [12]) is (1) PjBL relies on the problem to drive the curriculum. It is noted that the problem does not test the skills but assist in the development of the essential skill-sets to solve the problem. (2) The problem is typically open-structured which means that there is no one unique solution. New information is gathered and new skills are learned in an iterative manner. Perception of the problem, and thus the solution, gradually evolves and changes. (3) Students are gradually empowered to solve the problems and lecturers are simply coach or facilitate them. (4) Students are required to understand the problem. Many of the benefits of the application of $\mathrm{PjBL}$ have been shown in previous studies [13] [14] [15] [16].

\section{The prototype of Origami-Based Mathematics Instructional Materials.}

In this research, several projects have been developed to employ origami as learning media. This is inspired by the results of previous relevant research. First is the results found in Origami3 and Origami4 books. Both books are the result of the International Meeting of Origami, Science, and Education. In the Education section, there are many origami applications that are integrated into mathematical learning such as Using Triangular Boxes from Rectangular Paper to Enrich Trigonometry and Calculus [17], Fold paper and Enjoy Math: 
Origamics [18], Origametria: A Program to Teach Geometry and to Develop Learning Skills Using the Art of Origami [19], The Impact of Origami-Mathematics Lessons on Achievement and Spatial Ability of Middle-School Students [20],
Understanding the Effect of Origami Practice, Cognition, and Language on Spatial Reasoning, Modular Origami in the Secondary Geometry Classroom [22].

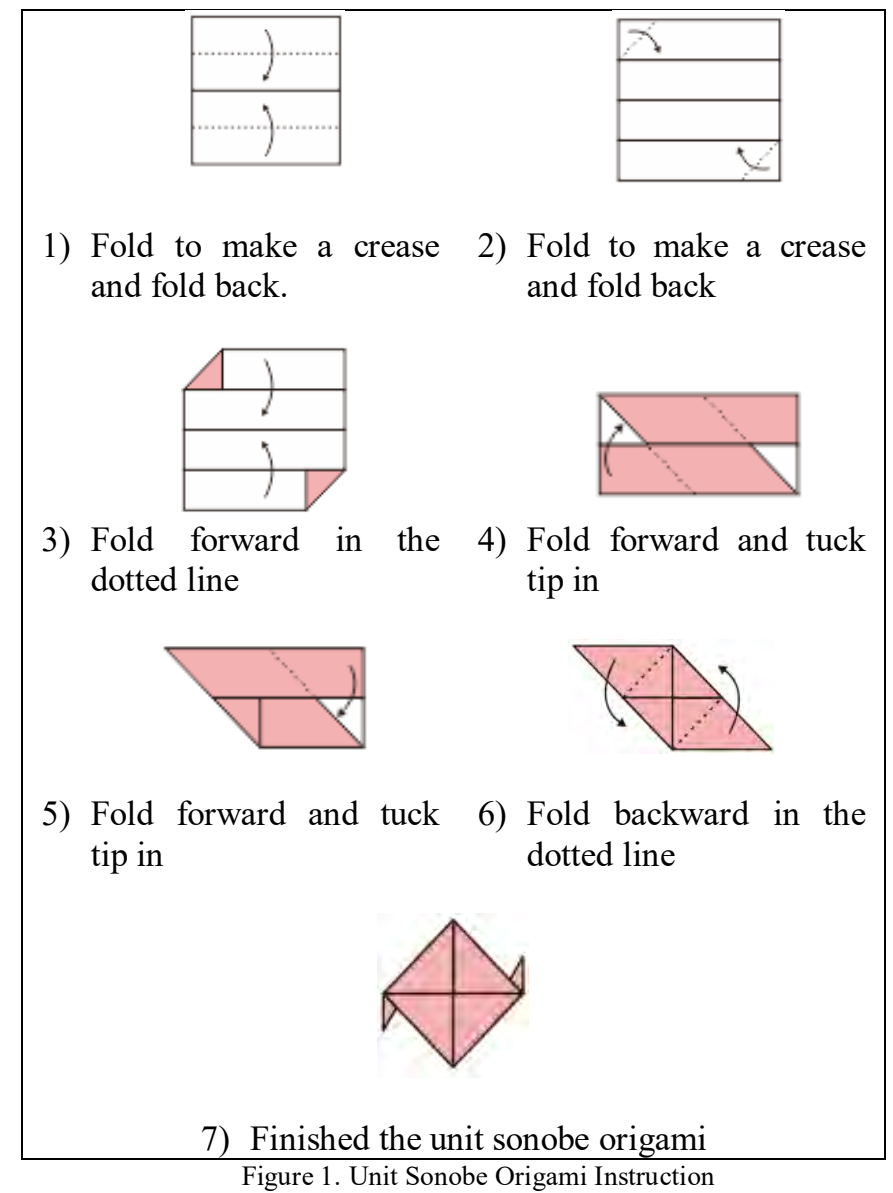

Figure 1. Unit Sonobe Origami Instruction

The second is a book written by Thomas Hull with Project Origami: Activities for Exploring Mathematics [23] which contains various learning activities by using origami that can be applied to a variety of topics. Based on these articles, researchers focused on origami development as a medium of learning mathematics in the form of Modular Origami, more specifically Sonobe Modular Origami with Project Based Learning approach.

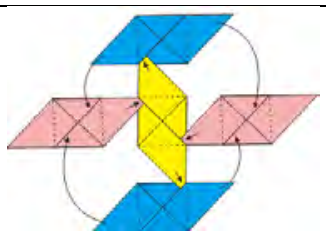

Make the six-unit of sonobe origami and connect them together

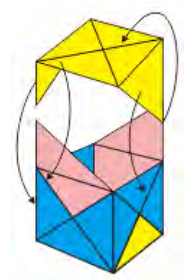

Tuck all part in
Sonobe Modular Origami is one of many Modular Origami. The steps for making the Sonobe Unit can be seen in Figure 1. Various forms can be created by combining the units of Sonobe origami. For example, a cube can be created using 6 units of Sonobe origami. A more complex shape is obtained by combining more units (see Figure 2).

Make the six-unit of sonobe origami and connect them together




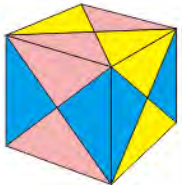

Finished

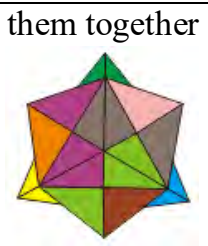

Finished a modular sonobe origami

Figure 2. Cube and Polyhedral using Sonobe Unit

The project developed in this study using Sonobe Modular Origami is Origami Puzzle Cube, Origami Sonobe Yoshimoto Cube, and Origami Modular Jewel. These projects can be applied to mathematics learning at the junior high school level. Referring to the activity developed by Thomas Hull, the arrangement of Project Instruction includes mathematical concepts (contains exploratory concepts), Synopsis (contains project overview), Content (contains explanations of mathematical content contained in the project), Handout (contains questions to explore mathematical concepts), and Time Allocation (contains the time required for project completion). In a complete manner, one of the projects can be seen in Appendix.

The first project is the Origami Puzzle Cube, this project asks students to make a sonobe cube, then wrap them into puzzle shapes. If the puzzles was put together it will be obtained a large cube with the size of $3 \times 3 \times 3$. The possible mathematical concepts that can be explored from the results of the student project are the comparison, surface area, and volume. Students can determine the surface area and volume of each puzzle, then look for a comparison of each puzzle against large cubes both in volume and width.

Next is Origami Sonobe Yoshimoto Cube, this project asks students to make 8 sonobe cube, then wrap them into a manipulative cube known as "sonobe Yoshimoto cube". In this cube, it is obtained an interesting form that is a combination of colors and other forms by changing it to a different shape. Possible explorations can be made to learn the mathematical concepts of fractional forms representing the color combinations contained in each form that can be manipulated. Furthermore, by measuring the length of the sides and the angle, the student can determine the congruence of the two field shapes found on the surface of the Yoshimoto cube sonobe. In addition, the Yoshimoto cube sonobe can serve as a substitute for the dice for the model of determining the opportunity subject.

The last is Origami Modular Jewel, this project asks students to assemble the origami sonobe units into modular origami shapes. Starting from a simple form by combining a minimum of sonobe units, with complex forms such as shown in Figure 2. The concept of explorable mathematics is that students determine the number of vertices and the number of ribs and the surface area of each shape. Furthermore, students can investigate whether the numbers obtained have certain patterns such as arithmetic or geometry series.

Lesson plans are needed for project-based learning to monitor learning progress from beginning to the end. A project is estimated to require 3 stages, the first stage is the preparation or planning that related to the project to be made including what materials are needed, the design that will be made, and the steps that must be done in making the project. The second stage is making the project, where students can communicate, negotiate, and collaborate to finish the project they had planned at the given time. The final stage is the presentation, where students present their work in front of other students and discuss it. This trains them to communicate their ideas. In addition, they can also learn to receive the feedback that they get from students or teachers.

The developed prototype was discussed with the junior high school teacher in the discussion group forum. Based on the results of discussions with two teachers of SMP namely Ibu Pipin and Mrs. Erwin related to the use of origami in the project and the projects developed, it is obtained the following results:

1. Media made from paper is very possible to be applied to the learning process because it does not require a high cost.

2. Origami can encourage students to perform activities independently and train students to make something neat and beautiful.

3. Projects can train students to plan, implement and present their own results.

4. The developed project of Origami can still be applied in mathematics learning process to explore other concepts, for example, Origami Puzzle Cube can be used to study the concept of algebraic terms. By designing a puzzle using two different colors and exemplifying colors as algebraic terms, it can be determined the algebraic form of each puzzle, as well as the sum of the algebraic tribes represented by the incorporation of the puzzles.

5. Activities to make projects can be done outside of the classroom or at home, where in the class, students focus for project progress consultation and presentation of project results.

Based on the results of the project development and discussion with the teacher, there are summaries of the project, form, and mathematical concepts that can be explored in Table 2.

The possible benefits other than those mentioned in the interview result are: Modular origami can support the mathematical classroom in many different pedagogical ways. It provides opportunities for cooperative groups, establishes unique mathematical experiences, builds connections between mathematics and the arts, teaches procedures, and provides unlimited extensions and permutations using the basic units discussed. For the teacher, the assessment is not difficult. Modular origami is an extendable activity with no deadlines for completion, providing immediate and direct feedback to the student, and is product-oriented [24]. Moreover about Projectbased learning, it has advantage such as students learning to be 
Self-reliant through planning and organizing, social learning to intrinsic motivation [25]. enhance collaboration skills, and differentiation providing

Table 2. Math Projects using Sonobe Origami

\begin{tabular}{|c|c|}
\hline No & Project \\
\hline 1 & Origami Puzzle Cube \\
\hline 2 & Origami Sonobe Yoshimoto Cube \\
\hline 3 & Origami Modular Jewel \\
\hline & \\
\hline
\end{tabular}

\section{IV.CONCLUSION}

Origami has a very varied form so it is very interesting to be made and studied. Based on the questionnaire and the textbook analysis, it is still very minimal utilization of origami in learning mathematics. Three projects developed in mathematics learning will be very interesting by using origami as a medium, especially Sonobe Modular Origami. Each project is able to explore more than one mathematical concept. This provides a tremendous opportunity for applying origami as a math learning medium in depth.

\section{ACKNOWLEDGMENT}

Thank you to Lembaga Penelitian dan Pengabdian kepada Masyarakat (LPPM) UMS which has funded this research through Competitive Regular Research grant (PEREKOM).

\section{REFERENCES}

[1] L. R. Aiken, "Two of Attitude Toward," J. Res. Math. Educ., vol. 5, no. 2, pp. 67-71, 2014.

[2] E. Peters et al., "Improving numeracy through values affirmation enhances decision and STEM outcomes," PLoS One, vol. 12, no. 7, pp. 1-19, 2017.

[3] T. Hull, "On the mathematics of flat origami," Congr. Numer., vol. 100, no. November 1995, pp. 215-224, 1994.

[4] H. Gur and M. Kobak-Demir, "Geometry Teaching via Origami: The Views of Secondary Mathematics Teacher Trainees," J. Educ. Pract., vol. 8, no. 15, pp. 65-71, 2017.

[5] S. Cakmak, M. Isiksal, and Y. Koc, "Investigating effect of origamibased instruction on elementary students spatial skills and perceptions," J. Educ. Res., vol. 107, no. 1, pp. 59-68, 2014.

[6] Y. N. Jati, "The Effect of Using Origami Paper to Teach the Perimeter of Plane Figures on Cognitive Achievement of Students Grade IX," A J. Lang. Lit. Cult. Educ., vol. 13, no. 1, pp. 35-42, 2017.

[7] N. Boakes, "Origami-Mathematics Lessons: Paper Folding as a Teaching Tool," vol. 1, pp. 1-9, 2008.

[8] N. J. Boakes, "Origami Instruction in the Middle School Mathematics Classroom: Its Impact on Spatial Visualization and Geometry Knowledge of Students," RMLE Online Vol. 32, No. 7, vol. 32, no. 7, pp. 1-12, 2009.

[9] J. W. Thomas, "A REVIEW OF RESEARCH ON PROJECT-BASED LEARNING," Buck Institute for Education, 2018. [Online]. Available: http://www.bobpearlman.org/BestPractices/PBL_Research.pdf.

[10] R. J. DeFillippi, "Introduction: Project-based Learning, Reflective Practices, and Learning Outcomes," SAGE Soc. Sci. Collect., vol. 23,
[11]

no. 1, pp. 5-10, 2001.

P. A. Ertmer and K. D. Glazewski, Essentials for $P B L$ implementation: Fostering collaboration, transforming roles, and scaffolding learning, no. 1980. West Lafayette: Purdue University Press, 2015.

[12] K. J. Chua, W. M. Yang, and H. L. Leo, "Enhanced and conventional project-based learning in an engineering design module," Int. $J$. Technol. Des. Educ., vol. 24, no. 4, pp. 437-458, 2014.

[13] I. Bilgin, Y. Karakuyu, and Y. Ay, "The effects of project-based learning on undergraduate students' achievement and self-efficacy beliefs towards science teaching," Eurasia J. Math. Sci. Technol. Educ., vol. 11, no. 3, pp. 469-477, 2015.

[14] Y. Gülbahar and H. Tinmaz, "Implementing project-based learning and E-portfolio assessment in an undergraduate course," J. Res. Technol. Educ., vol. 38, no. 3, pp. 309-327, 2006.

[15] S. Han, R. Capraro, and M. M. Capraro, "How Science, Technology, Engineering, and Mathematics (Stem) Project-Based Learning (Pbl) Affects High, Middle, and Low Achievers Differently: the Impact of Student Factors on Achievement," Int. J. Sci. Math. Educ., vol. 13, no. 5, pp. 1089-1113, 2015.

[16] M. Shin, "Effects of Project-based Learning on Students' Motivation and Self-efficacy," Teach. English, vol. 73, no. 1, pp. 95-115, 2018.

[17] V'Ann Cornelius and Arnold Tubis, "Using Triangular Boxes from Rectangular Paper to Enrich Trigonometry and Calculus", in Origami3: Third International Meeting of Origami Science, Math, and Education /Thomas Hull, editor, Canada: A.K Pieters, 2002, p.299306

[18]Kazuo Haga, "Fold Paper and Enjoy Math: Origamics", in Origami3: Third International Meeting of Origami Science, Math, and Education /Thomas Hull, editor, Canada: A.K Pieters, 2002, p.307-328

[19] Miri Golan and Paul Jackson, "Origametria: A Program to Teach Geometry and to Develop Learning Skills Using the Art of Origami", in Origami4: Fourth International Meeting of Origami Science, Mathematics, and Education/Robert J. Lang, editor, USA: A.K Pieters, 2009, p.459-470

[20] Norma J. Boakes, "The Impact of Origami-Mathematics Lessons on Achievement and Spatial Ability of Middle-School Students", in Origami4: Fourth International Meeting of Origami Science, Mathematics, and Education/Robert J. Lang, editor, USA: A.K Pieters, 2009, p.471-482

[21]Michael Wilson, Robin Flanagan, Rona Gurkewitz, and Laura Skrip, "Understanding the Effect of Origami Practice, Cognition, and Language on Spatial Reasoning", in Origami4: Fourth International Meeting of Origami Science, Mathematics, and Education/Robert J. Lang, editor,USA: A.K Pieters, 2009, p.483-496

[22]Margaret Cagle, "Modular Origami in the Secondary Geometry Classroom", in Origami4: Fourth International Meeting of Origami Science, Mathematics, and Education/Robert J. Lang, editor, USA: A.K Pieters, 2009, p.497-506 
[23]Thomas Hull, Project Origami Activities for Exploring Mathematics, 2nd ed., US: CRC Press, 2013

[24] Wenciker, Brock, and Patrick Flynn. "Modular Origami in the Mathematics Classroom." Bridges: Mathematical Connections in Art,
Music, and Science. Bridges Conference, 2004.

[25]Bell, Stephanie. "Project-based learning for the 21 st century: Skills for the future." The Clearing House 83.2, 2010: 39-43. 